\title{
Impact of a comprehensive antimicrobial stewardship program on antimicrobial utilization and clinical isolates distribution in ICUs
}

\section{Jiaojiao Song}

Zhejiang University School of Medicine Second Affiliated Hospital

\section{Rongsheng Zhu}

Zhejiang University School of Medicine Second Affiliated Hospital

\section{Leiqing Li}

Zhejiang University School of Medicine Second Affiliated Hospital

\section{Lingcheng $\mathrm{Xu}$}

Zhejiang University School of Medicine Second Affiliated Hospital

\section{Quan Zhou}

Zhejiang University School of Medicine Second Affiliated Hospital

\section{Donghang $\mathrm{Xu}$}

Zhejiang University School of Medicine Second Affiliated Hospital

\section{Yangmin Hu}

Zhejiang University School of Medicine Second Affiliated Hospital

\section{Xuanding Wang ( $\nabla$ xudwang@zju.edu.cn)}

Zhejiang University School of Medicine Second Affiliated Hospital https://orcid.org/0000-0001-59915887

\section{Research}

Keywords: antimicrobials, antimicrobial stewardship, antimicrobial utilization, distribution, intensive care unit

Posted Date: January 13th, 2020

DOl: https://doi.org/10.21203/rs.2.20643/v1

License: (1) (1) This work is licensed under a Creative Commons Attribution 4.0 International License. Read Full License 


\section{Abstract}

Objective

This study aimed to evaluate the effect of a comprehensive antimicrobial stewardship program (ASP) and provide clinical evidence for the scientific stewardship of antimicrobials in intensive care units (ICUs) of a teaching hospital.

Methods

Between January 2013 and December 2018, we conducted a prospective study, based on an antimicrobial computerized clinical decision support system (aCDSS) deployed in 2015 in ICUs of a tertiary and teaching hospital. The primary outcomes included initial and overall use prevalence of antimicrobials. The second outcomes were the detection rate of common clinical isolates before and after therapeutic antimicrobial use, and the change in patterns of resistance of 5 common clinical isolates in the ICU.

Results

Various types of broad-spectrum antimicrobial use prevalence continued to increase from 2013 to 2015 , since 2016, where initial use of carbapenems and glycopeptides were counterbalanced by an increase in use of the first/second-generation cephalosporins, $\beta$-lactam and $\beta$-lactamase inhibitor combinations and linezolid. From 2015 to 2018, the proportion of extended-broad spectrum antimicrobials alone, widecoverage therapy and combination therapy decreased significantly $(P<0.05)$. Similarly, where use of carbapenems, glycopeptides, third/fourth-generation cephalosporins and anti-fungi agents were counterbalanced by an increase in overall use of the first/second-generation cephalosporins and $\beta$ lactam and $\beta$-lactamase inhibitor combinations. A total of 21891 strains of bacteria and fungi were detected in ICUs from 2015 to 2018, of them, 6.5\% (1426/21891) strains were detected before antimicrobial treatment. The detection proportion of Staphylococcus aureus , Escherichia coli, Klebsiella pneumoniae and fastidious bacteria were significantly higher before antimicrobial treatment $(P<0.05)$, while Acinetobacter baumannii , Burkholderia cepacia , and Candida spp were significantly lower in all non-repetitive clinical isolates $(P<0.05)$.

Conclusions

The implementation of a comprehensive ASP combining CDSS in ICUs seems to be effective to improve outcomes on antimicrobial utilization and clinical isolates distribution in critically ill patients.

\section{Introduction}

Antimicrobial resistance (AMR) is a global public health threat, especially in hospitalized patients with substantial health and economic consequences. The choice of antimicrobials for the treatment of multidrug-resistant organism (MDRO) infections have become increasingly limited and expensive[1, 2]. 
Inappropriate or sub-optimal utilization of antimicrobials over the past few decades has driven increases in rates of resistance, with the consequent effects of worse patient outcomes[3], undermining various aspects of health care and creating new disease burden[4,5]. Thus, optimizing antimicrobial therapy is of great importance since the link between inappropriate use of antimicrobials and the emergence of AMR has been well established, particularly in intensive care units (ICUs) where broad-spectrum antimicrobials are frequently prescribed $[6,7]$ and it turns out that $30 \%-60 \%$ of them are unnecessary, inappropriate, or suboptimal[8-11].

Antimicrobial stewardship (AMS) involves a multifaceted approach to optimize antimicrobial use, minimize unintended consequences and combat the emergence of AMR[12-17]. The guidelines for the implementation of antimicrobial stewardship program (ASP) were published in 2007 and updated in 2016 by the American Society of Infectious Diseases (IDSA) and the Society for Healthcare Epidemiology of America (SHEA) [18, 19]. Although ASP in China strived to improve rational use of antimicrobials and indeed achieved some success, gaps still existed[20], especially in the ICU where patients often needed urgent effective treatment while antimicrobial resistance had been identified as a serious problem[21], the obstacles to AMS were significant in this vulnerable and complex patient population[22]. Point prevalence surveys (PPS) in 53 countries around the world showed that only $20 \%$ of adults hospitalized patients receiving therapeutic antimicrobials were infected with identified pathogens[23], targeted antimicrobial treatment is facing difficulties all over the world. Considering that the use of antimicrobial agents can affect the distribution and drug resistance of clinical isolates in medical institutions significantly[24-26], timely acquisitions of appropriate microbiological specimens before antimicrobial use is critical[27]. Clinical decision support system (CDSS), as one of the components of ASPs, serving to integrate multiple variables and allow physicians to make more appropriate treatment decisions. Computerized systems appear to be logical choices to maintain ASP competence and have become increasingly appealing because of the growing migration of other clinical systems to computerized platforms.

We developed and deployed an interactive, web-based computerized antimicrobial clinical decision support system (aCDSS), allowing and assisting stewards to obtain more reliable microbial outcomes on distribution and resistance patterns of clinical isolates and assess antimicrobial use, providing facilitylevel epidemiological data and illustrated trends in antimicrobial use. Insufficient studies describing the long-lasting impact of ASP in ICUs in a real-life setting have been documented so far[28-32], thus, our primary objective of this investigation was to describe the implementation and the effects of a comprehensive ASP focusing on CDSS in ICUs of our hospital during 6 consecutive years.

\section{Materials And Methods}

\section{Design and Setting}

A 6-year prospective study was conducted at the Second Affiliated Hospital of Zhejiang University School of Medicine (SAHZU), a tertiary care and Joint Commission International (JCl) accredited hospital, with 
133-bed combined ICUs. During the study period from January 1, 2013 to December 31, 2018, all patients admitted to ICU for more than 24 hours and prescribed antimicrobials were eligible for inclusion.

\section{Interventions}

Hospital-wide AMS strategies had been implemented under the guidance of a cross-sectoral committee consisting of infectious-disease-trained physicians, infection control practitioners, pharmacists, and clinical microbiologists. Our ASP was employed since Jan. 2015 which is a bundle of interventions including basic management measures and strengthening interventions aimed to facilitate the appropriate use of antimicrobials and combat AMR in health care settings. Besides, our ICUs established robust infection prevention and control initiatives to curtail onwards transmission of AMR microbes[33], and practices did not change appreciably during the study period.

Basic strategies focused on feedback \& monitoring, coupled with weekly review and multi-disciplinary team discussion of antimicrobial regimens in randomly selected patients. Feedback was delivered by quarterly reporting of aggregate microbiological data in ICUs. In addition, mandate hospital-wide educational modules were made on principles of appropriate antimicrobial use, strategies of related policies and the mechanisms of AMR, for all healthcare professionals involved in antimicrobial ordering, administration and monitoring through online and cased-based learning.

Prior to the study, SAHZU had adopted an electronic medical record system (EMRS) integrated with computerized physician order entry (CPOE) system which allowed submitting medication, laboratory, and radiology orders electronically. In nursing care, a mobile device was used for bedside medication and laboratory orders reconciliation. Data on the timing of medication administration and microbiological specimen sampling were routinely recorded by the device, which was later transferred and stored in the nursing recording System (NRS). We began to develop the aCDSS, a web-based administrative database in late 2012. It was embedded in the EMRS and could access the hospital information system (HIS), EMRS, NRS, laboratory information system (LIS), pharmacy information system (PIS), surgical anesthesia information management system (AIMS) and human resources system (HRS) in real time based on HL-7 standard. It was applicable to all clinical departments since January 2015 to facilitate and streamline ASP through the following strengthening interventions:

i. Hierarchical management of antimicrobials: when prescribed antimicrobials through the $\mathrm{CPOE}$, the aCDSS was triggered to enter the authorization interface and had established prescription authority for providers at all levels. Physicians must be familiar with their prescription authority and when issued a non-authorized antimicrobial drug order, it would be sent to qualified personnel and could only be prescribed when audited and permitted. The medical department regularly organized standardized training on the antimicrobial agent management regulations and clinical application knowledge for all physicians and pharmacists. After trained and qualified, the corresponding prescription authority could be obtained. The members of hospital infection control department and antimicrobial prescription review group regularly checked and provided feedback on the physicians' 
prescription authorization, the use of broad-spectrum antimicrobials, and the consistency of the indications and the actual situation of the patients, etc., so as to urge continuous improvement;

ii. Pre-audit of indications for broad-spectrum antimicrobials: when prescribed a broad-spectrum antimicrobial agent including $\beta$-lactam and $\beta$-lactamase inhibitor combinations, carbapenems, tigecycline and so on, EMRS would pop up an electronic application form for the use of the corresponding antimicrobial agent after the prescription authority was passed, then the prescriber was asked to select the preset indication according to the actual situation of the patient before submitting the medical order (see Table 1). Stewardship rounds were conducted in ICUs, if there was any difficulty or an antimicrobial prescription modification was recommended by the ASP team, a face-to-face conference was held between team members and attending intensivists to decide the ultimate treatment;

iii. Online clinical decision support: all the diagnostic testing results were communicated to healthcare providers and provided interpretive expertise by the system in real time, assisting prescribers to review antimicrobial prescriptions and help to make preliminary recommendations based on infectious diseases resources;

iv. Intervening in combination therapy: verification and confirmation of indications for combination therapy were provided when prescribed two or more antimicrobials (see Table 2).

v. Standardizing microbiological specimen sampling practice: the aCDSS ensured timely microbiological specimen sampling for microbial culture before therapeutic antibiotic use in inpatients. When ordered antimicrobials through the $\mathrm{CPOE}$, the system automatically detected patient's surgery information through accessing EMRS. If no surgery information was identified, the aCDSS judged the ordered antibiotic as therapeutic use; otherwise, prescribers should choose the intended indication. In the next step, prescribers were requested to select the infection site on the software interface. The aCDSS detected patient's data on microbiological specimen sampling time through accessing NRS. In cases no data were identified, the prescriber could only quit the aCDSS, submit a laboratory order for bacterial culture through the $\mathrm{CPOE}$, then obtain the specimen and scan the barcode on the specimen to generate the sampling data (see Figure 1). 
1. The clinical isolates are only sensitive to the same class of antimicrobials (note the exclusion of contaminated or accompanying strain)

1. The currently used target antimicrobial was ineffective, and exclude other factors that lead to ineffective antimicrobial therapy

1. Initial empiric therapy for severe infections (must be used after qualified microbiological specimens sampling)

Bloodstream infections (sepsis, etc.)

Accompanied by respiratory failure, shock, DIC and other complications

Acute peritonitis caused by perforation of organs, acute pelvic inflammatory disease, etc.

Severe pneumonia caused by reflux aspiration

In suspicious of Gram-negative bacilli infection or first considered severe infection of the hepatobiliary system

Serious mixed infections

Severe burns, severe combined injuries, multiple injuries, etc.

Severe infection of Gram-negative bacilli in the central nervous system

Severe complicated abdominal infection after abdominal surgery

1. Infection in immunosuppressive patients

Long-term treatment with immunosuppressive agents

Receiving chemotherapy

Receiving high-dose corticosteroids for more than 10 days

White blood cell count $\leq 1000 / \mathrm{mm}^{3}$ for more than one week

The absolute value of neutrophils is $\leq 500 / \mathrm{mm}^{3}$ for more than one week.

The number of CD4+ T lymphocytes in HIV-infected patients is $\leq 400 / \mathrm{mm}^{3}$

Congenital immune deficiency

After splenectomy

\section{Other choices}

Recommended by authoritative guides

Recommended by multidisciplinary discussion

Recommended by a physician with the corresponding antimicrobial prescription authorization

\section{Table 1}

The schematic table of electronic application form of carbapenems.

DIC: disseminated intravascular coagulation; HIV: acquired immunodeficiency syndrome 


\section{Indications of combination therapy}

1. Serious infections in immunodeficient patients (such as significant reduction in white blood cell count, long-term high-dose use of glucocorticoids, splenectomy, AIDS, etc.)

1. Two or more pathogens that cannot be controlled by a single antimicrobial agent (one of the pathogens cannot be covered by the currently used drug)

1. Severe infections such as severe pneumonia, infective endocarditis, or sepsis that cannot be controlled by a single antimicrobial agent

1. MDRO infection that cannot be effectively controlled by a single antimicrobial agent, especially hospital acquired infection

1. Synergistic effects of combination therapy can reduce the dose of a single antimicrobial agent, thereby reducing adverse reactions

1. Long-term treatment, but pathogens are susceptible to certain MDRO infections, such as tuberculosis

1. A serious life-threatening infection that has not been identified by the pathogen

1. According to the authoritative guidelines' recommendations

Table 2

The schematic table of indications of combination therapy.

AIDS: acquired immunodeficiency syndrome; MDRO: multidrug-resistant organism

\section{Data Collection and Outcome Assessment}

The aCDSS consolidated data on patient-specific demographics, surgery information, antimicrobials prescribed and related administration time, microbiological specimen sampling time, microbial culture results from EMRS, HIS, NRS and LIS (see Figure 2). Resistant rate of 5 common bacteria including Staphylococcus aureus, Escherichia coli, Klebsiella pneumoniae, Pseudomonas aeruginosa, and Acinetobacter baumannii was defined as the percentage of isolates resistant to selected antimicrobials. Duplicated isolates cultured from the same specimen from the same inpatient were not included for analysis. Antimicrobial use prevalence was calculated by dividing the number of cases prescribed specific antimicrobial over the total number of cases in the defined period. Antimicrobial regimens were analyzed within the initial $24 \mathrm{~h}$ and throughout the whole ICU stay.

The primary outcomes included: (i) initial and overall use prevalence of antimicrobials. The second outcome measured for this study were: (i) the detection rate of common clinical isolates before and after therapeutic antimicrobial use; and (ii) the change in patterns of resistance of 5 common clinical isolates in the ICU.

\section{Statistical analysis}


Data were expressed as the number (\%) for categorical variables. Comparisons were performed by chisquare test or Fisher's exact test for categorical variables, as appropriate. All analyses were performed with SPSS version 20.0 (SPSS Inc., Chicago, IL, USA). A two-tailed P value of $<0.05$ was used as a threshold of a significant difference for all statistical tests.

\section{Results}

\section{Primary outcomes}

Various types of broad-spectrum antimicrobial use prevalence continued to increase from 2013 to 2015, since 2016, where initial use of carbapenems and glycopeptides were counterbalanced by an increase in use of the first/second-generation cephalosporins, $\beta$-lactam and $\beta$-lactamase inhibitor combinations and linezolid, which called "squeezing the balloon"[34] (see Table 3). Further analysis showed that from 2015 to 2018 , the proportion of extended-broad spectrum antimicrobials alone, wide-coverage therapy and combination therapy decreased significantly $(P<0.05)$, the maximum percentage of declines were $63.4 \%$, $13.9 \%$ and $42.9 \%$, respectively (see Fig. 3 ). Similarly, where use of carbapenems, glycopeptides, third/fourth-generation cephalosporins and anti-fungi agents were counterbalanced by an increase in overall use of the first/second-generation cephalosporins and $\beta$-lactam and $\beta$-lactamase inhibitor combinations (see Table 4).

Table 3

Changes of initial antimicrobial use in ICUs during the study period

\begin{tabular}{|c|c|c|c|c|c|c|}
\hline Antimicrobials/prevelance & $\begin{array}{l}2013(n \\
=862)\end{array}$ & $\begin{array}{l}2014(n \\
=841)\end{array}$ & $\begin{array}{l}2015 \\
(n= \\
970)\end{array}$ & $\begin{array}{l}2016(n \\
=887)\end{array}$ & $\begin{array}{l}2017(n \\
=1279)\end{array}$ & $\begin{array}{l}2018(\mathrm{n} \\
=1326)\end{array}$ \\
\hline $\begin{array}{l}\beta \text {-lactam and } \beta \text {-lactamase } \\
\text { inhibitor combinations }\end{array}$ & $42.0 \%$ & $43.2 \%$ & $40.3 \%$ & $38.8 \%$ & $42.1 \%$ & $47.6 \%$ \\
\hline Carbapenems & $28.4 \%$ & $32.9 \%$ & $42.9 \%$ & $42.5 \%$ & $27.9 \%$ & $19.8 \%$ \\
\hline Glycopeptides & $10.3 \%$ & $10.3 \%$ & $8.1 \%$ & $10.4 \%$ & $5.8 \%$ & $5.1 \%$ \\
\hline Linezolid & $4.2 \%$ & $2.4 \%$ & $3.9 \%$ & $5.3 \%$ & $4.5 \%$ & $5.1 \%$ \\
\hline $\begin{array}{l}\text { First/Second-generation } \\
\text { cephalosporins }\end{array}$ & $3.0 \%$ & $3.7 \%$ & $3.4 \%$ & $3.6 \%$ & $7.7 \%$ & $15.5 \%$ \\
\hline $\begin{array}{l}\text { Third/Fourth-generation } \\
\text { Cephalosporins }\end{array}$ & $12.9 \%$ & $8.6 \%$ & $4.8 \%$ & $3.6 \%$ & $9.7 \%$ & $5.7 \%$ \\
\hline Tigecycline & $0.9 \%$ & $0.6 \%$ & $2.3 \%$ & $2.8 \%$ & $2.3 \%$ & $1.6 \%$ \\
\hline Antifungal drugs & $3.4 \%$ & $3.7 \%$ & $4.6 \%$ & $5.9 \%$ & $3.4 \%$ & $4.1 \%$ \\
\hline
\end{tabular}


Table 4

Changes of overall antimicrobial use in ICUs during the study period

\begin{tabular}{|c|c|c|c|c|c|c|}
\hline Antimicrobials/prevelance & $\begin{array}{l}2013 \\
(n= \\
867)\end{array}$ & $\begin{array}{l}2014(n \\
=912)\end{array}$ & $\begin{array}{l}2015 \\
(n= \\
1085)\end{array}$ & $\begin{array}{l}2016 \\
(n= \\
965)\end{array}$ & $\begin{array}{l}2017(n \\
=1312)\end{array}$ & $\begin{array}{l}2018(\mathrm{n} \\
=1352)\end{array}$ \\
\hline $\begin{array}{l}\beta \text {-lactam and } \beta \text {-lactamase } \\
\text { inhibitor combinations }\end{array}$ & $64.7 \%$ & $66.1 \%$ & $57.8 \%$ & $58.0 \%$ & $64.4 \%$ & $71.7 \%$ \\
\hline Carbapenems & $60.0 \%$ & $66.3 \%$ & $78.5 \%$ & $73.0 \%$ & $59.2 \%$ & $49.9 \%$ \\
\hline Glycopeptides & $40.6 \%$ & $40.2 \%$ & $33.6 \%$ & $36.0 \%$ & $26.3 \%$ & $23.1 \%$ \\
\hline Linezolid & $19.0 \%$ & $11.4 \%$ & $20.6 \%$ & $17.2 \%$ & $18.1 \%$ & $21.6 \%$ \\
\hline $\begin{array}{l}\text { First/Second-generation } \\
\text { cephalosporins }\end{array}$ & $5.1 \%$ & $7.1 \%$ & $7.9 \%$ & $6.6 \%$ & $11.7 \%$ & $20.2 \%$ \\
\hline $\begin{array}{l}\text { Third/Fourth-generation } \\
\text { Cephalosporins }\end{array}$ & $24.0 \%$ & $14.6 \%$ & $10.1 \%$ & $6.9 \%$ & $12.7 \%$ & $8.3 \%$ \\
\hline Tigecycline & $6.9 \%$ & $13.3 \%$ & $25.9 \%$ & $25.0 \%$ & $20.5 \%$ & $16.0 \%$ \\
\hline Anti-fungal agents & $18.5 \%$ & $20.5 \%$ & $24.5 \%$ & $23.8 \%$ & $18.8 \%$ & $15.8 \%$ \\
\hline
\end{tabular}

\section{The Second outcomes}

In 2015-2018, a total of 21891 strains of non-repetitive strains were detected in ICUs, including 4521 strains of Gram-positive bacteria (20.6\%), 15252 strains of Gram-negative bacteria (69.7\%), and 2118 strains of fungi (9.7\%). 1426 strains were detected before antimicrobial treatment, accounting for only $6.5 \%$ of the total number of non-repetitive strains at the same period. The detection rate of Staphylococcus aureus, Escherichia coli, Klebsiella pneumoniae, fastidious bacteria such as Streptococcus, Haemophilus influenzae and Moraxella catarrhalis were significantly higher before antimicrobial treatment $(P<0.05)$, while Acinetobacter baumannii, Burkholderia cepacia, and Candida spp were significantly lower $(P<0.05)$ (see Fig. 4).

The antimicrobial resistance profiles of 5 major clinical isolates in 4 consecutive years showed that in addition to Pseudomonas aeruginosa, the resistant rates of strains detected before antimicrobial treatment including Staphylococcus aureus, Escherichia coli, Klebsiella pneumoniae, and Acinetobacter baumannii were significantly lower $(P<0.05)$, more so in Klebsiella pneumoniae, which even reduced by approximately $2 / 3$ (see Table 5). 
Table 5

Comparison of antimicrobial resistance of 5 major isolates before versus after initiation of therapeutic antimicrobial use in 2015-2018

\begin{tabular}{|c|c|c|c|}
\hline Isolates/Antibiotics & Before & After & All \\
\hline Staphylococcus aureus & 186 & 1269 & 1455 \\
\hline Penicillin G & $89.8 \%$ & $95.1 \%$ & $94.4 \%$ \\
\hline MRSA & $37.2 \%$ & $63.6 \%$ & $60.2 \%$ \\
\hline Erythromycin & $54.0 \%$ & $65.8 \%$ & $64.2 \%^{a}$ \\
\hline Clindamycin & $46.5 \%$ & $58.6 \%$ & $57.1 \%$ \\
\hline Tetracycline & $26.9 \%$ & $44.0 \%$ & $41.8 \%$ \\
\hline Gentamicin & $5.4 \%$ & $12.6 \%$ & $11.7 \%$ \\
\hline Ciprofloxacin & $20.2 \%$ & $51.8 \%$ & $47.6 \%$ \\
\hline Levofloxacin & $19.8 \%$ & $53.4 \%$ & $49.1 \%$ \\
\hline SMZ-TMP & $7.0 \%$ & $5.1 \%$ & $29.5 \%$ \\
\hline Vancomycin & $0.0 \%$ & $0.0 \%$ & $0.0 \%$ \\
\hline Escherichia coli & 112 & 435 & 547 \\
\hline Amoxicillin clavulanic acid & $8.8 \%$ & $17.2 \%$ & $15.5 \%$ \\
\hline Cefazolin & $54.5 \%$ & $66.9 \%$ & $64.4 \%$ \\
\hline Ceftriaxone & $49.0 \%$ & $59.3 \%$ & $57.0 \%$ \\
\hline Ceftazidime & $14.7 \%$ & $27.3 \%$ & $24.6 \%$ \\
\hline Cefepime & $14.3 \%$ & $20.5 \%$ & $19.2 \%$ \\
\hline Aztreonam & $31.3 \%$ & $41.1 \%$ & $39.1 \%$ \\
\hline Cefoperazone sulbactam & $4.9 \%$ & $12.2 \%$ & $10.6 \%$ \\
\hline Piperacillin-tazobactam & $6.3 \%$ & $7.4 \%$ & $7.1 \%$ \\
\hline Imipenem & $5.4 \%$ & $5.3 \%$ & $5.4 \%$ \\
\hline Meropenem & $3.8 \%$ & $5.5 \%$ & $5.2 \%$ \\
\hline Gentamicin & $30.0 \%$ & $38.3 \%$ & $36.5 \%$ \\
\hline
\end{tabular}




\begin{tabular}{|c|c|c|c|}
\hline Isolates/Antibiotics & Before & After & All \\
\hline Amikacin & $2.7 \%$ & $6.0 \%$ & $5.3 \%$ \\
\hline Ciprofloxacin & $41.1 \%$ & $47.7 \%$ & $46.3 \%$ \\
\hline Levofloxacin & $39.3 \%$ & $45.0 \%$ & $43.9 \%$ \\
\hline Tigecycline & $0.0 \%$ & $0.7 \%$ & $0.6 \%$ \\
\hline Polymyxin & $0.0 \%$ & $1.5 \%$ & $1.3 \%$ \\
\hline ESBL+ & $48.9 \%$ & $55.0 \%$ & $53.7 \%$ \\
\hline Klebsiella pneumoniae & 346 & 3716 & 4062 \\
\hline Amoxicillin clavulanic acid & $21.4 \%$ & $66.2 \%$ & $62.3 \% 9$ \\
\hline Cefazolin & $24.8 \%$ & $71.0 \%$ & $67.0 \%$ \\
\hline Cefoxitin & $20.1 \%$ & $64.3 \%$ & $60.5 \% 9$ \\
\hline Ceftriaxone & $24.5 \%$ & $69.0 \%$ & $65.1 \% 9$ \\
\hline Ceftazidime & $19.3 \%$ & $67.4 \%$ & $63.1 \%$ \\
\hline Cefepime & $19.4 \%$ & $60.8 \%$ & $57.2 \%$ \\
\hline Aztreonam & $24.3 \%$ & $67.8 \%$ & $64.1 \%$ \\
\hline Cefoperazone sulbactam & $21.0 \%$ & $65.2 \%$ & $61.5 \%$ \\
\hline Piperacillin-tazobactam & $18.8 \%$ & $63.3 \%$ & $59.5 \%$ \\
\hline Ertapenem & $11.1 \%$ & $51.6 \%$ & $47.4 \%$ \\
\hline Imipenem & $19.5 \%$ & $63.1 \%$ & $59.4 \%$ \\
\hline Meropenem & $20.3 \%$ & $63.8 \%$ & $60.0 \%$ \\
\hline Gentamicin & $16.0 \%$ & $45.8 \%$ & $43.2 \%$ \\
\hline Tobramycin & $13.0 \%$ & $39.3 \%$ & $37.1 \%$ \\
\hline Amikacin & $10.7 \%$ & $35.2 \%$ & $33.1 \%$ \\
\hline Ciprofloxacin & $19.9 \%$ & $59.7 \%$ & $56.3 \%$ \\
\hline Levofloxacin & $18.8 \%$ & $57.1 \%$ & $53.9 \%$ \\
\hline
\end{tabular}




\begin{tabular}{|c|c|c|c|}
\hline Isolates/Antibiotics & Before & After & All \\
\hline SMZ-TMP & $16.3 \%$ & $44.1 \%$ & $41.8 \% 9$ \\
\hline Furanolide & $35.2 \%$ & $67.0 \%$ & $64.5 \% 9$ \\
\hline Tigecycline & $5.8 \%$ & $16.1 \%$ & $15.2 \%$ \\
\hline Pseudomonas aeruginosa & 150 & 2239 & 2389 \\
\hline Cefoperazone sulbactam & $38.6 \%$ & $41.8 \%$ & $41.6 \%$ \\
\hline Piperacillin-tazobactam & $29.7 \%$ & $35.3 \%$ & $35.0 \%$ \\
\hline Cefepime & $29.5 \%$ & $37.6 \%$ & $37.1 \%$ \\
\hline Aztreonam & $39.4 \%$ & $49.3 \%$ & $48.8 \%$ \\
\hline Imipenem & $52.1 \%$ & $58.3 \%$ & $57.9 \%$ \\
\hline Meropenem & $48.5 \%$ & $50.6 \%$ & $50.5 \%$ \\
\hline Gentamicin & $10.1 \%$ & $9.8 \%$ & $9.8 \%$ \\
\hline Amikacin & $8.7 \%$ & $7.5 \%$ & $7.6 \%$ \\
\hline Ciprofloxacin & $26.0 \%$ & $33.5 \%$ & $33.0 \%$ \\
\hline Levofloxacin & $26.0 \%$ & $33.0 \%$ & $32.5 \%$ \\
\hline Polymyxin & $0.0 \%$ & $1.2 \%$ & $1.2 \%$ \\
\hline Acinetobacter baumannii & 231 & 4798 & 5033 \\
\hline Ceftriaxone & $74.4 \%$ & $88.8 \%$ & $88.1 \% 9$ \\
\hline Cefepime & $73.2 \%$ & $88.5 \%$ & $87.8 \%{ }^{9}$ \\
\hline Aztreonam & $92.5 \%$ & $95.8 \%$ & $95.6 \%$ \\
\hline Cefoperazone sulbactam & $43.0 \%$ & $53.1 \%$ & $52.6 \%$ \\
\hline Piperacillin-tazobactam & $69.8 \%$ & $86.3 \%$ & $85.6 \%$ \\
\hline Imipenem & $73.9 \%$ & $89.0 \%$ & $88.3 \% 9$ \\
\hline Meropenem & $74.7 \%$ & $89.1 \%$ & $88.4 \%^{9}$ \\
\hline Gentamicin & $55.6 \%$ & $69.9 \%$ & $69.2 \% 9$ \\
\hline
\end{tabular}

9, detection rate of strains before antimicrobial treatment compared with all the non-repetitive strains, $\mathrm{P}<0.05$. 


\begin{tabular}{|llll|}
\hline Isolates/Antibiotics & Before & After & All \\
\hline Ciprofloxacin & $75.2 \%$ & $89.4 \%$ & $88.7 \%$ \\
\hline Levofloxacin & $49.4 \%$ & $60.7 \%$ & $60.2 \%$ \\
\hline Tigecycline & $6.9 \%$ & $7.7 \%$ & $7.6 \%$ \\
\hline Polymyxin & $1.2 \%$ & $1.0 \%$ & $1.0 \%$ \\
\hline $\begin{array}{l}\text { 9, detection rate of strains before antimicrobial treatment compared with all the non-repetitive strains, } \\
\text { P<0.05. }\end{array}$ \\
\hline
\end{tabular}

\section{Discussion}

This study, over a 6-year period and in a real-life setting, analyzed the impact of antimicrobial use on microbial outcomes, along with a comprehensive ASP focusing on aCDSS, feedback \& monitoring, weekly review of antimicrobials and multidisciplinary team discussion of antimicrobial regimens and education on antimicrobial use. Measuring the impact of an ASP is crucial to maintain administrative support and we provided real-world experience from established ASPs in critical care settings. The aCDSS consists of several components including intervention in microbiological specimen sampling practice, hierarchical stewardship, education resources, and antimicrobial prescription recommendations. The design of this practice-based study was not to specifically measure the impact of any individual initiative but rather an integrated program in critical care.

We used data from academic hospital ICUs covering 6 years. As one of the wards with highest antimicrobial consumption, ICU is a gathering place for immunocompromised patients, and the nosocomial infection prevalence is much higher due to wide-spread use of broad-spectrum antimicrobials, glucocorticoids, immunosuppressive agents, and various invasive procedures than that of the ordinary wards. An international PPS indicates that $51 \%$ of the 1,265 ICUs in 75 countries around the world have infections identified and $71 \%$ are prescribed antimicrobials[35]. On one hand, a prompt institution of effective antimicrobial regimens for proper coverage of causative pathogens is vital in critically ill patients and involves physicians with infectious disease expertise considering the complexity of decision-making process; On the other hand, an indiscriminate use of broad-spectrum antimicrobials is closely contributed to mortality[36, 37], with higher AMR burden[38]. In this regard, timely and accurate retention of microbiological specimens to obtain appropriate cultures before antimicrobial administration is the basis for targeted therapy, obtaining more reliable local antimicrobial susceptibility patterns to increase the likelihood of prescribing appropriate initial antimicrobials, especially for patients with a higher risk of death in patients in the ICU. Our study has demonstrated that the introduction of an antimicrobial decision support tool into the ICU, ensuring the pre-conditions of microbiological specimens sampling before therapeutic antimicrobials use, was associated with measurable improvements in both initial antimicrobial use and overall use. Given that it has long been recognized that the inability to easily get access on data on local antimicrobial use patterns can be a gap[39-41], standardized metrics to 
individual facilities across a healthcare system has been a major advance. CDSS linked to EMRS and other clinical systems to this computerized platform has been proved to have the potential to facilitate the dissemination of information to intensivists for optimal use in therapeutic decision-making[42-46]. We consider all antimicrobial prescribing important and have shown that it is feasible to make full use of web-based application to help work with intensivists to improve antimicrobial prescribing practice by considering all patients' accompanying microbiology diagnostic testing issues helping the interpretation of some of our results and encouraging evidence-based decisions regarding choice of therapy. Our study achieved significantly reduced use of carbapenems, glycopeptides, third/fourth-generation cephalosporins and anti-fungi agents in all.

Our study has a number of limitations. Firstly, it is an observational study in a single center without randomization and thus is subject to known biases. Secondly, many confounding factors interfere with the judgment of AMS performance. For example, rapid diagnostic test plays a collaborative role for ASPs[47]. At last, this study occurred in academic ICUs, most of the patients admitted to our ICUs were not "first-hand" patients who had been prescribed antimicrobials in community medical institutions or outpatient clinics before, thus, the microbiological data obtained through aCDSS was already partially biased, Furthermore, academic ICUs differ from community-based ICUs or long-term nursing care institutions in terms of staffing models and admitting more complex patients receiving broader spectrum antimicrobials. This study only represents the efforts of an established ASP with appropriate resourcing for wed-based interventions and may not be generalizable to other centers. In summary, while we were able to show temporal improvements in antimicrobial utilization structure concerning our interventions, larger, appropriately conducted randomized controlled trials or at least a controlled quasi-experimental design with longitudinal time series analysis are needed to further evaluate the effects of ASP including CDSS in critical care settings[48, 49].

\section{Conclusions}

Computer-assisted decision support was associated with more reliable surveillance data on microbiology and sustained improvements in antimicrobials use in critical care. Institution of a comprehensive antimicrobial stewardship program combing clinical decision support system has the potential to be an effective means to improve clinical diagnosis and treatment and to optimize antimicrobial use.

\section{Declarations}

\section{Ethics approval}

All procedures were performed in accordance with the Declaration of Helsinki of the World Medical Association approved by the Ethics Committee of the Second Affiliated Hospital of Zhejiang University

\section{Consent for publication}

Not applicable 
Availability of data and materials

The datasets during and/or analysed during the current study available from the corresponding author on reasonable request.

\section{Competing interests}

There is no financial and non-financi ompeting interests.

\section{Authors' contributions}

Xuanding Wang: Work design; Jiaojiao Song: data selection; Leiqing Li, Lingcheng Xu: data analysis; Quan Zhou, Donghang Xu: manuscript writing

\section{Funding}

This work was supported by the Joint Construction Project of National Health and Family Planning Commission and Zhejiang Province (grant number WKJ2014-2-011) and Zhejiang Provincial Public Welfare Technology Application Research Project (grant number 2015C33107).

\section{Acknowledgements}

We thank the assistance provided by members of the microbiology laboratory, information technologists and pharmacists in the Second Affiliated Hospital of Zhejiang University School of Medicine.

\section{References}

1. Quadri F, Mazer-Amirshahi M, Fox ER, Hawley KL, Pines JM, Zocchi MS, May L: Antibacterial drug shortages from 2001 to 2013: implications for clinical practice. Clinical infectious diseases : an official publication of the Infectious Diseases Society of America 2015, 60(12):1737-1742.

2. Leung E, Weil DE, Raviglione M, Nakatani H: The WHO policy package to combat antimicrobial resistance. Bulletin of the World Health Organization 2011, 89(5):390-392.

3. MacKenzie FM, Struelens MJ, Towner KJ, Gould IM: Report of the Consensus Conference on Antibiotic Resistance; Prevention and Control (ARPAC). Clinical microbiology and infection : the official publication of the European Society of Clinical Microbiology and Infectious Diseases 2005, 11(11):938-954.

4. Laxminarayan R: Antibiotic effectiveness: balancing conservation against innovation. Science (New York, NY) 2014, 345(6202):1299-1301.

5. Laxminarayan R, Duse A, Wattal C, Zaidi AK, Wertheim HF, Sumpradit N, Vlieghe E, Hara GL, Gould IM, Goossens $\mathrm{H}$ et al: Antibiotic resistance-the need for global solutions. The Lancet Infectious diseases 2013, 13(12):1057-1098. 
6. Armand-Lefevre L, Angebault C, Barbier F, Hamelet E, Defrance G, Ruppe E, Bronchard R, Lepeule R, Lucet JC, El Mniai A et al: Emergence of imipenem-resistant gram-negative bacilli in intestinal flora of intensive care patients. Antimicrobial agents and chemotherapy 2013, 57(3):1488-1495.

7. Bass SN, Bauer SR, Neuner EA, Lam SW: Impact of combination antimicrobial therapy on mortality risk for critically ill patients with carbapenem-resistant bacteremia. Antimicrobial agents and chemotherapy 2015, 59(7):3748-3753.

8. Bergmans DC, Bonten MJ, Gaillard CA, van Tiel FH, van der Geest S, de Leeuw PW, Stobberingh EE: Indications for antibiotic use in ICU patients: a one-year prospective surveillance. J Antimicrob Chemother 1997, 39(4):527-535.

9. Roberts JA, Paul SK, Akova M, Bassetti M, De Waele JJ, Dimopoulos G, Kaukonen KM, Koulenti D, Martin C, Montravers $\mathrm{P}$ et al: DALI: defining antibiotic levels in intensive care unit patients: are current beta-lactam antibiotic doses sufficient for critically ill patients? Clinical infectious diseases : an official publication of the Infectious Diseases Society of America 2014, 58(8):1072-1083.

10. Kollef $\mathrm{MH}$ : Optimizing antibiotic therapy in the intensive care unit setting. Critical care (London, England) 2001, 5(4):189-195.

11. Kollef MH, Fraser VJ: Antibiotic resistance in the intensive care unit. Annals of internal medicine 2001, 134(4):298-314.

12. Ramasethu J, Kawakita T: Antibiotic stewardship in perinatal and neonatal care. Seminars in fetal \& neonatal medicine 2017, 22(5):278-283.

13. Cantey JB, Wozniak PS, Pruszynski JE, Sanchez PJ: Reducing unnecessary antibiotic use in the neonatal intensive care unit (SCOUT): a prospective interrupted time-series study. The Lancet Infectious diseases 2016, 16(10):1178-1184.

14. Manzoni P, Dall'Agnola A: Reducing unnecessary antibiotic exposure in preterm neonates: an achievable goal. The Lancet Infectious diseases 2016, 16(10):1094-1096.

15. Ting JY, Synnes A, Roberts A, Deshpandey A, Dow K, Yoon EW, Lee KS, Dobson S, Lee SK, Shah PS: Association Between Antibiotic Use and Neonatal Mortality and Morbidities in Very Low-Birth-Weight Infants Without Culture-Proven Sepsis or Necrotizing Enterocolitis. JAMA pediatrics 2016, 170(12):1181-1187.

16. Nzegwu NI, Rychalsky MR, Nallu LA, Song X, Deng Y, Natusch AM, Baltimore RS, Paci GR, Bizzarro MJ: Implementation of an Antimicrobial Stewardship Program in a Neonatal Intensive Care Unit. Infection control and hospital epidemiology 2017, 38(10):1137-1143.

17. Rodriguez-Bano J, Perez-Moreno MA, Penalva G, Garnacho-Montero J, Pinto C, Salcedo I, FernandezUrrusuno R, Neth O, Gil-Navarro MV, Perez-Milena A et al: Outcomes of the PIRASOA programme, an antimicrobial stewardship programme implemented in hospitals of the public Health system of Andalusia, Spain: an ecological study of time-trend analysis. Clinical microbiology and infection : the official publication of the European Society of Clinical Microbiology and Infectious Diseases 2019.

18. Dellit TH, Owens RC, McGowan JE, Jr., Gerding DN, Weinstein RA, Burke JP, Huskins WC, Paterson DL, Fishman NO, Carpenter CF et al: Infectious Diseases Society of America and the Society for 
Healthcare Epidemiology of America guidelines for developing an institutional program to enhance antimicrobial stewardship. Clinical infectious diseases : an official publication of the Infectious Diseases Society of America 2007, 44(2):159-177.

19. Barlam TF, Cosgrove SE, Abbo LM, MacDougall C, Schuetz AN, Septimus EJ, Srinivasan A, Dellit TH, Falck-Ytter YT, Fishman NO et al: Implementing an Antibiotic Stewardship Program: Guidelines by the Infectious Diseases Society of America and the Society for Healthcare Epidemiology of America. Clinical infectious diseases : an official publication of the Infectious Diseases Society of America 2016, 62(10):e51-77.

20. Xiao Y: Antimicrobial Stewardship in China: Systems, Actions and Future Strategies. Clinical infectious diseases : an official publication of the Infectious Diseases Society of America 2018, 67(suppl_2):S135-s141.

21. Brusselaers $\mathrm{N}$, Vogelaers $\mathrm{D}, \mathrm{Blot} \mathrm{S}$ : The rising problem of antimicrobial resistance in the intensive care unit. Annals of intensive care 2011, 1:47.

22. Lawrence $\mathrm{KL}$, Kollef $\mathrm{MH}$ : Antimicrobial stewardship in the intensive care unit: advances and obstacles. American journal of respiratory and critical care medicine 2009, 179(6):434-438.

23. Versporten A, Zarb P, Caniaux I, Gros MF, Drapier N, Miller M, Jarlier V, Nathwani D, Goossens H: Antimicrobial consumption and resistance in adult hospital inpatients in $\mathbf{5 3}$ countries: results of an internet-based global point prevalence survey. Lancet Glob Health 2018, 6(6):e619-e629.

24. Al-Mayahi M, Cian A, Lipsky BA, Suva D, Muller C, Landelle C, Miozzari HH, Uckay I: Administration of antibiotic agents before intraoperative sampling in orthopedic infections alters culture results. The Journal of infection 2015, 71(5):518-525.

25. Wunderink RG, Waterer GW: Appropriate microbiological testing in community-acquired pneumonia. Chest 2001, 119(1):5-7.

26. Kanegaye JT, Soliemanzadeh P, Bradley JS: Lumbar puncture in pediatric bacterial meningitis: defining the time interval for recovery of cerebrospinal fluid pathogens after parenteral antibiotic pretreatment. Pediatrics 2001, 108(5):1169-1174.

27. Organization WH: Diagnostic stewardship: a guide to implementation in antimicrobial resistance surveillance sites. 2016.

28. Rimawi RH, Mazer MA, Siraj DS, Gooch M, Cook PP: Impact of regular collaboration between infectious diseases and critical care practitioners on antimicrobial utilization and patient outcome. Critical care medicine 2013, 41(9):2099-2107.

29. Zhang $Y Z$, Singh S: Antibiotic stewardship programmes in intensive care units: Why, how, and where are they leading us. World journal of critical care medicine 2015, 4(1):13-28.

30. Hou D, Wang Q, Jiang C, Tian C, Li H, Ji B: Evaluation of the short-term effects of antimicrobial stewardship in the intensive care unit at a tertiary hospital in China. PloS one 2014, 9(7):e101447.

31. Hurford A, Morris AM, Fisman DN, Wu J: Linking antimicrobial prescribing to antimicrobial resistance in the ICU: before and after an antimicrobial stewardship program. Epidemics 2012, 4(4):203-210. 
32. Maechler F, Schwab F, Geffers C, Meyer E, Leistner R, Gastmeier P: Antibiotic stewardship in Germany: a cross-sectional questionnaire survey of 355 intensive care units. Infection 2014, 42(1):119-125.

33. Holmes AH, Moore LS, Sundsfjord A, Steinbakk M, Regmi S, Karkey A, Guerin PJ, Piddock LJ: Understanding the mechanisms and drivers of antimicrobial resistance. Lancet (London, England) 2016, 387(10014):176-187.

34. Burke JP: Antibiotic resistance-squeezing the balloon? Jama 1998, 280(14):1270-1271.

35. Vincent JL, Rello J, Marshall J, Silva E, Anzueto A, Martin CD, Moreno R, Lipman J, Gomersall C, Sakr $Y$ et al: International study of the prevalence and outcomes of infection in intensive care units. Jama 2009, 302(21):2323-2329.

36. Kumar A, Ellis P, Arabi Y, Roberts D, Light B, Parrillo JE, Dodek P, Wood G, Kumar A, Simon D et al: Initiation of inappropriate antimicrobial therapy results in a fivefold reduction of survival in human septic shock. Chest 2009, 136(5):1237-1248.

37. Levy MM, Evans LE, Rhodes A: The Surviving Sepsis Campaign Bundle: 2018 update. Intensive care medicine 2018, 44(6):925-928.

38. Figueiredo Costa S: Impact of antimicrobial resistance on the treatment and outcome of patients with sepsis. Shock (Augusta, Ga) 2008, 30 Suppl 1:23-29.

39. Howard P, Pulcini C, Levy Hara G, West RM, Gould IM, Harbarth S, Nathwani D: An international crosssectional survey of antimicrobial stewardship programmes in hospitals. J Antimicrob Chemother 2015, 70(4):1245-1255.

40. Abbo L, Lo K, Sinkowitz-Cochran R, Burke AC, Hopkins RS, Srinivasan A, Hooton TM: Antimicrobial stewardship programs in Florida's acute care facilities. Infection control and hospital epidemiology 2013, 34(6):634-637.

41. Johannsson B, Beekmann SE, Srinivasan A, Hersh AL, Laxminarayan R, Polgreen PM: Improving antimicrobial stewardship: the evolution of programmatic strategies and barriers. Infection control and hospital epidemiology 2011, 32(4):367-374.

42. Pestotnik SL, Classen DC, Evans RS, Burke JP: Implementing antibiotic practice guidelines through computer-assisted decision support: clinical and financial outcomes. Annals of internal medicine 1996, 124(10):884-890.

43. Kullar R, Goff DA, Schulz LT, Fox BC, Rose WE: The "epic" challenge of optimizing antimicrobial stewardship: the role of electronic medical records and technology. Clinical infectious diseases : an official publication of the Infectious Diseases Society of America 2013, 57(7):1005-1013.

44. Leibovici L, Gitelman V, Yehezkelli Y, Poznanski O, Milo G, Paul M, Ein-Dor P: Improving empirical antibiotic treatment: prospective, nonintervention testing of a decision support system. Journal of internal medicine 1997, 242(5):395-400.

45. Forrest GN, Van Schooneveld TC, Kullar R, Schulz LT, Duong P, Postelnick M: Use of electronic health records and clinical decision support systems for antimicrobial stewardship. Clinical infectious 
diseases : an official publication of the Infectious Diseases Society of America 2014, 59 Suppl 3:S122-133.

46. Arboe B, Laub RR, Kronborg G, Knudsen JD: Evaluation of the decision support system for antimicrobial treatment, TREAT, in an acute medical ward of a university hospital. International journal of infectious diseases : IJID : official publication of the International Society for Infectious Diseases 2014, 29:156-161.

47. Caliendo AM, Gilbert DN, Ginocchio CC, Hanson KE, May L, Quinn TC, Tenover FC, Alland D, Blaschke AJ, Bonomo RA et al: Better tests, better care: improved diagnostics for infectious diseases. Clinical infectious diseases : an official publication of the Infectious Diseases Society of America 2013, 57 Suppl 3:S139-170.

48. Johnston ME, Langton KB, Haynes RB, Mathieu A: Effects of computer-based clinical decision support systems on clinician performance and patient outcome. A critical appraisal of research. Annals of internal medicine 1994, 120(2):135-142.

49. Ramsay C, Brown E, Hartman G, Davey P: Room for improvement: a systematic review of the quality of evaluations of interventions to improve hospital antibiotic prescribing. $J$ Antimicrob Chemother 2003, 52(5):764-771.

\section{Figures}

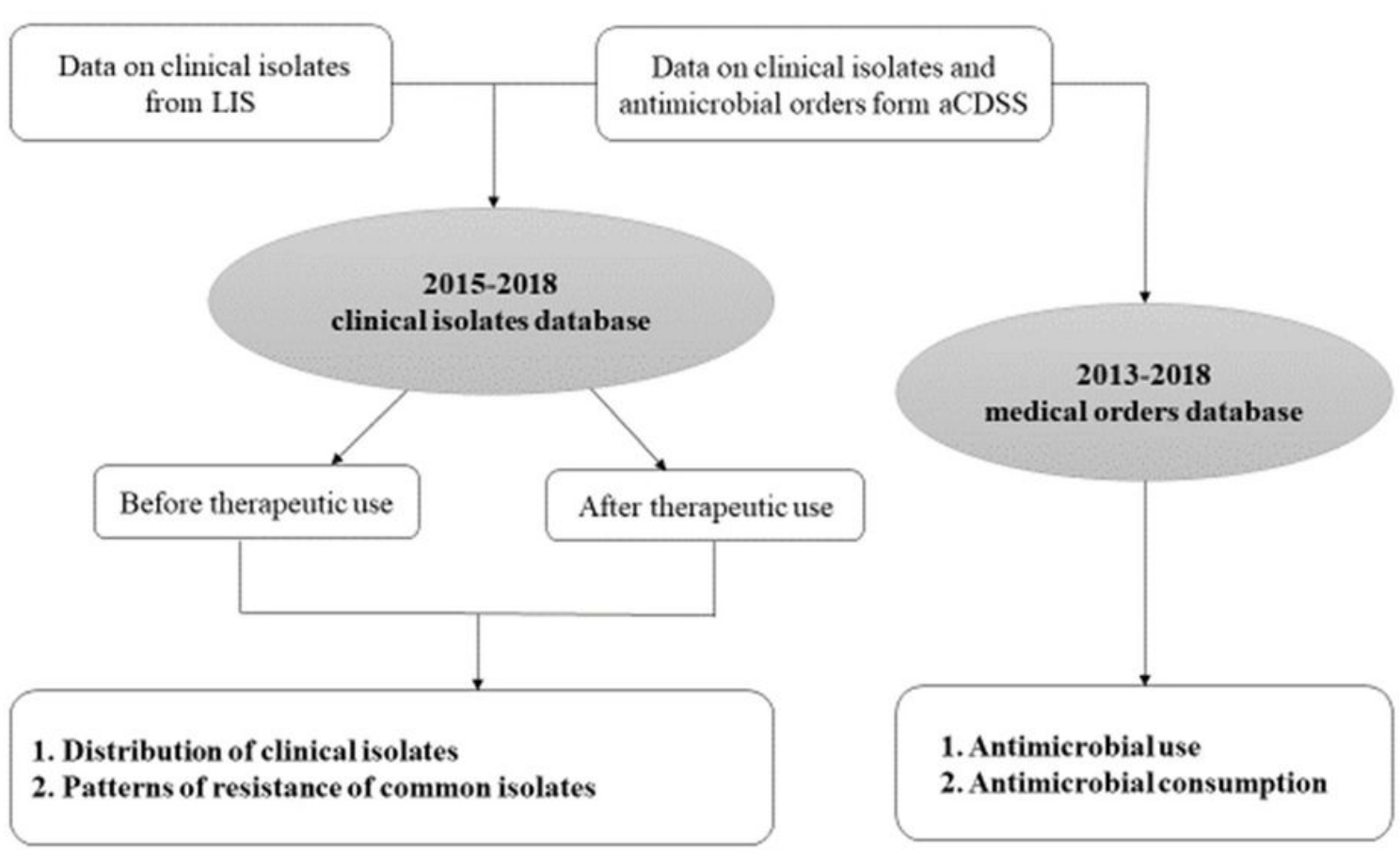

Figure 1 
Flow charts of the intervention in microbiological specimen sampling. a. In cases sampling was unnecessary (treatment of syphilis), no appropriate specimen was available (i.e., deep organ infection), or the pathogen had already been identified earlier, the antibiotic prescription was allowed in the absence of sampling data. Specified reasons should be submitted in the system's structured menu for evaluation and feedback. aCDSS: computerized antimicrobial clinical decision support system; CPOE: computerized physician order entry

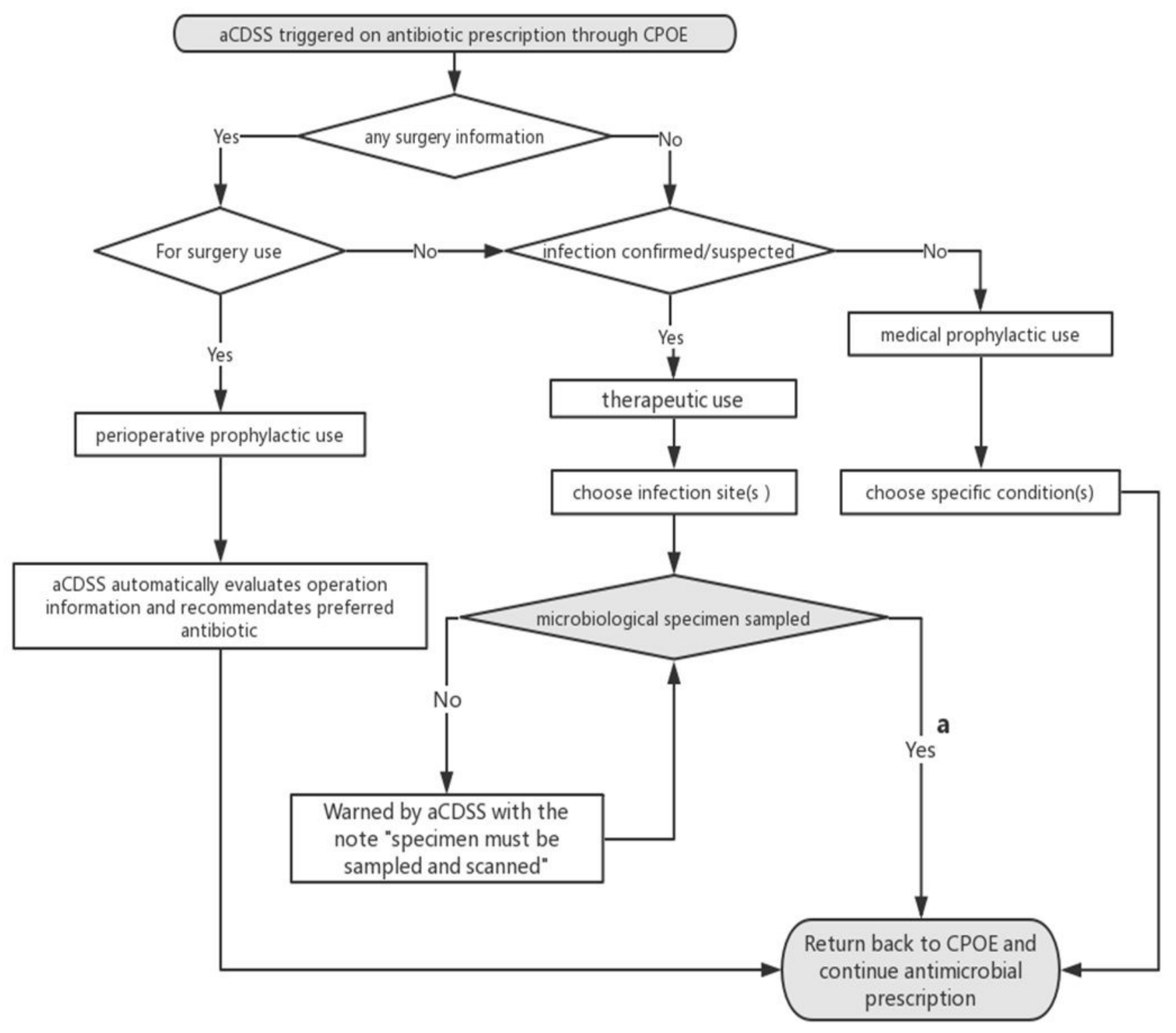

Figure 2

Flow charts of data collection and study outcomes 


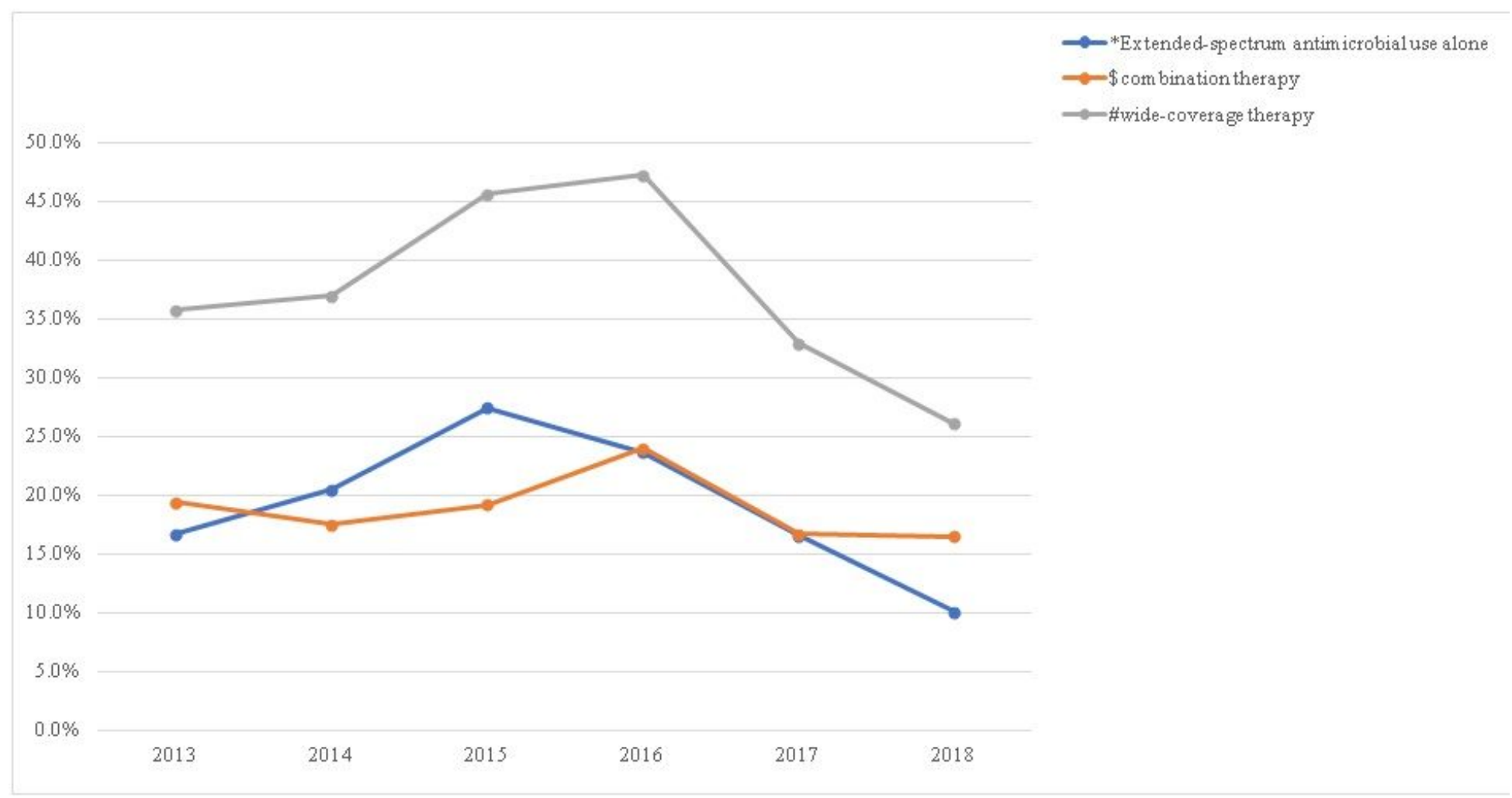

\section{Figure 3}

Changes of medication structure in initial 24h in ICUs from 2015-2018 *:

carbapenem/tigecycline/polymyxin use alone; \$: two or more kinds of antimicrobial agents used at the same time; \#: broad-spectrum combination therapy or carbapenem (except ertapenem), tigecycline, polymyxin use alone 


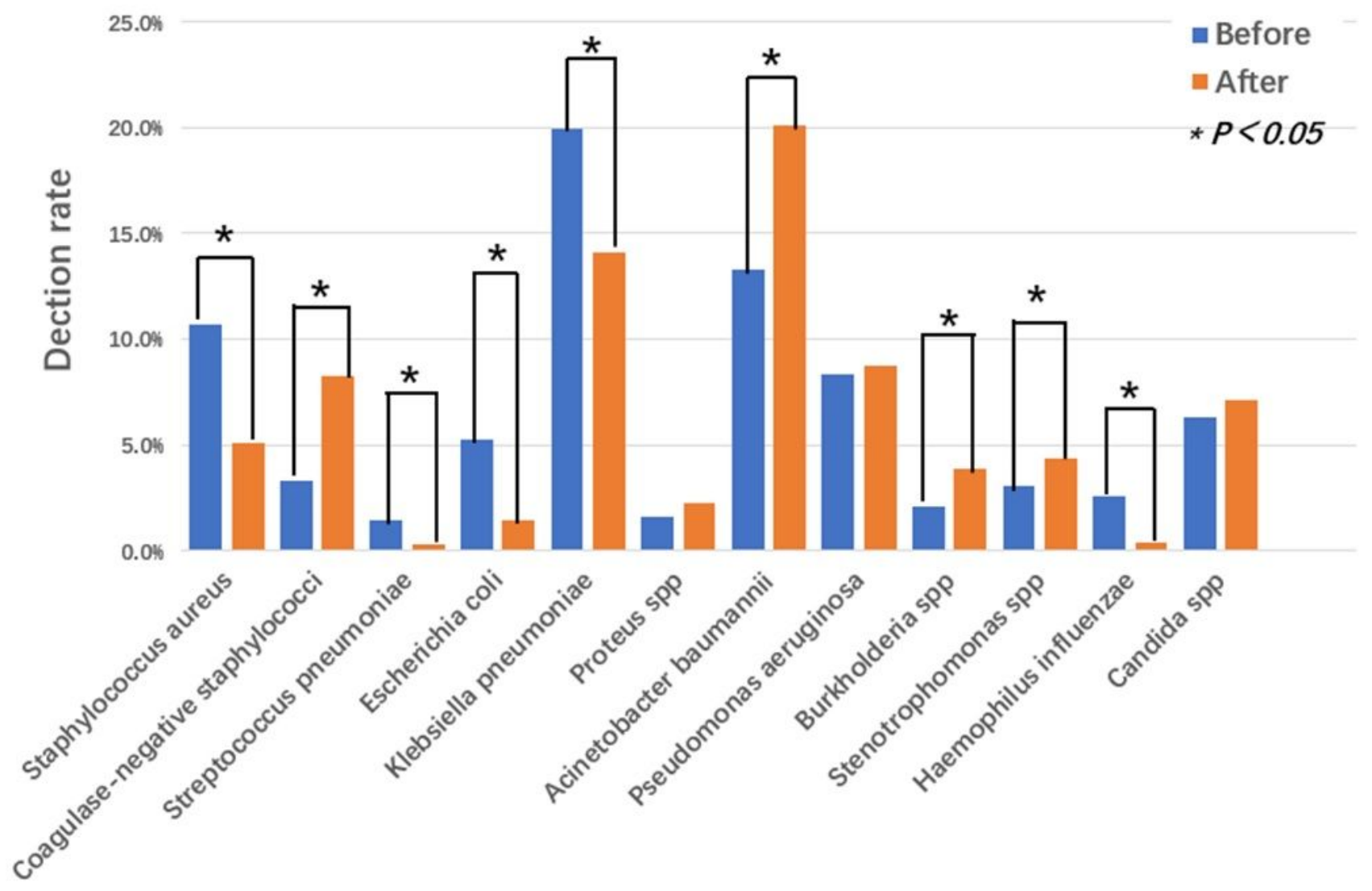

Figure 4

Detection rate of common clinical isolates before versus after initiation of therapeutic antimicrobial use in 2015-2018 